International Mathematical Forum, 2, 2007, no. 47, 2333 - 2338

\title{
Existence of Infinitely Many Brake Orbits of Lagrangian Autonomous Systems on Tori ${ }^{1}$
}

\author{
Guangcun Lu \\ Department of Mathematics \\ Beijing Normal University \\ Beijing 100875, P. R. China \\ gclu@bnu.edu.cn \\ MingYan Wang \\ Department of Mathematics \\ Beijing Normal University \\ Beijing 100875, P. R. China \\ wangmingyan@mail.bnu.edu.cn
}

\begin{abstract}
In this note we point out that the arguments by Campos and Tarallo [1] implied the existence of periodic brake orbits with minimal period greater than any prescribed number for real analytic Lagrangian autonomous systems on tori.
\end{abstract}

Mathematics Subject Classification: 58E05; 70H05; 34C25

Keywords: Brake orbit, Lagrangian systems

An autonomous Lagrangian system on the Riemannian torus $\left(\mathbb{T}^{n}=\mathbb{R}^{n} / \mathbb{Z}^{n}, A\right)$ with a potential $V$ is described on $\mathbb{R}^{n}$ by the equation

$$
\frac{d}{d t}\left(\frac{\partial L}{\partial \dot{q}}\right)-\frac{\partial L}{\partial q}=0
$$

\footnotetext{
${ }^{1}$ Partially supported by the NNSF 10371007 and 10671017 of China and the Program for New Century Excellent Talents of the Education Ministry of China.
} 
where $L(q, \dot{q})=\frac{1}{2}(A(q) \dot{q}, \dot{q})-V(q)$, the matrix $A(q)$ is symmetric and positive definite for every $q \in \mathbb{R}^{n}$ and $A$ and $V$ are 1-period in each variable. For the flat metric $A$, i.e. $A$ being a positive matrix on $\mathbb{R}^{n}$ without depending on $q \in \mathbb{R}^{n}$, and $C^{3}$-smooth $V$, Yiming Long and the first author proved in [2] that for any $\tau>0$ the system (1) possesses infinitely many geometrically distinct contractible periodic solution orbits of periods being integer multiples of $\tau$. When both $A$ and $V$ are assumed to be real analytic functions of $q$, it was also proved in [1] by Campos and Tarallo that for every $\tau_{0}>0$ there exists a periodic solution to (1) with minimal period greater than $\tau_{0}$. Here we are interested in the existence of $\tau$-periodic solutions $q: \mathbb{R} \rightarrow \mathbb{R}^{n}$ of (1) satisfying

$$
\dot{q}(0)=\dot{q}(\tau / 2)=0
$$

Such periodic solutions are called brake orbits of (1) because they bear some formal analogy with brakes studied by Seifert [4]. Clearly, a $C^{1}$-map $q: \mathbb{R} \rightarrow$ $\mathbb{R}^{n}$ with

$$
q(\tau / 2+t)=q(\tau / 2-t) \forall t \in \mathbb{R}
$$

must satisfy (2). Moreover, a $\tau$-periodic map $q: \mathbb{R} \rightarrow \mathbb{R}^{n}$ satisfies (3) if and only if

$$
q(t)=q(-t) \forall t \in \mathbb{R} .
$$

A map $q: \mathbb{R} \rightarrow \mathbb{R}^{n}$ satisfying (4) is said to be reversible in time. So a periodic solution of (1) which is reversible in time gives rise to a brake orbit of (1). Note also that a $\tau$-periodic map $q: \mathbb{R} \rightarrow \mathbb{R}^{n}$ satisfies (4) if and only if $q(k \tau / 2+t)=q(k \tau / 2-t)$ for any $t \in \mathbb{R}$ and $k \in \mathbb{Z}$. Thus a $\tau$-periodic solution $q: \mathbb{R} \rightarrow \mathbb{R}^{n}$ of (1) satisfying $q(t)=q(-t)$ automatically products many other such periodic solutions of $(1)$, such as $q_{k}(t)=q(t+k \tau / 2)+h$ with $k \in \mathbb{Z}$ and $h \in \mathbb{Z}^{n}$. For $\tau_{i}$-periodic solutions $q_{i}: \mathbb{R} \rightarrow \mathbb{R}^{n}$ of (1) being reversible in time, $i=1,2$, they are called distinct if one of them cannot be obtained from another in the way above. Suitably modifying the arguments in [1] we get

Theorem 1. For real analytic $A$ and $V$, if $V$ is also nonconstant then for every $\tau>0$ there exists a reversible period solution of (1) with minimal period greater than $\tau$, and thus there exist brake orbits of (1) with minimal period greater than any prescribed number.

As in Theorem 2 of [1], in the case that $A$ and $V$ are only smooth, if $V$ is also nonconstant then there exist uncountable number of distinct and nonconstant reversible period solutions of (1). Let $H$ be the Hilbert space of the absolutely continuous 1 -periodic function $q: \mathbb{R} \rightarrow \mathbb{R}^{n}$ with $L^{2}$-derivative on $[0,1]$. There 
is a natural $\mathbb{Z}^{n}$-action on it. For each $\tau>0$, a $\tau$-periodic solution $q$ to (1) corresponds to a critical point $u$ of the $\mathbb{Z}^{n}$-invariant smooth functional

$$
\mathcal{A}_{\tau}(u)=\frac{1}{2 \tau^{2}} \int_{0}^{1}(A(u(t)) \dot{u}(t), \dot{u}(t)) d t-\int_{0}^{1} V(u(t)) d t
$$

on $H$ via $u(t):=q(\tau t)$ (cf. [1]). Denote by $H_{0}$ the subspace of $H$ consisting of those $u \in H$ which are reversible in time, i.e., $u(t)=u(-t)$ for any $t \in \mathbb{R}$. It is easily checked that $H_{0}$ is a Hilbert subspace of $H$. As in [3], one can prove that $\mathcal{A}_{\tau}$ also satisfies the Palais-Smale condition on $\widetilde{H}_{0}:=H_{0} / \mathbb{Z}^{n}$.

Lemma 2. A map $q: \mathbb{R} \rightarrow \mathbb{R}^{n}$ which is reversible in time is a $\tau$-periodic solution to (1) if and only if $u(t):=q(\tau t)$ is a critical point of $\mathcal{A}_{\tau}$ on $H_{0}$.

Proof. We only need to prove that a critical point $u_{0}$ of $\mathcal{A}_{\tau}$ on $H_{0}$ is also one of $\mathcal{A}_{\tau}$ on $H$. Let $\Xi: H \rightarrow H$ be the Hilbert space isomorphism defined by $u \rightarrow \Xi(u), \Xi(u)(t)=u(-t)$. It is actually involution with fixed point set $\operatorname{Fix}(\Xi)=H_{0}$. One easily checks that $\mathcal{A}_{\tau}(\Xi(u))=\mathcal{A}_{\tau}(u)$ for any $u \in H$. It follows that $\Xi\left(\mathcal{A}_{\tau}^{\prime}(u)\right)=\mathcal{A}_{\tau}^{\prime}(\Xi(u))$ for any $u \in H$. Consider the orthogonal decomposition $\mathcal{A}_{\tau}^{\prime}(u)=\mathcal{A}_{\tau}^{\prime}(u)_{0} \oplus \mathcal{A}_{\tau}^{\prime}(u)_{0}^{\perp} \in H_{0} \oplus H_{0}^{\perp}$. Since $\Xi\left(u_{0}\right)=u_{0}$ and $\Xi\left(\mathcal{A}_{\tau}^{\prime}\left(u_{0}\right)_{0}\right)=\mathcal{A}_{\tau}^{\prime}\left(u_{0}\right)_{0}$, we deduce that $\Xi\left(\mathcal{A}_{\tau}^{\prime}\left(u_{0}\right)_{0}^{\perp}\right)=\mathcal{A}_{\tau}^{\prime}\left(u_{0}\right)_{0}^{\perp}$ and thus $\mathcal{A}_{\tau}^{\prime}\left(u_{0}\right)_{0}^{\perp}=0$. Hence $\mathcal{A}_{\tau}^{\prime}\left(u_{0}\right)=\mathcal{A}_{\tau}^{\prime}\left(u_{0}\right)_{0} \in H_{0}$. By the definition,

$$
\left(\left(\left.\mathcal{A}_{\tau}\right|_{H_{0}}\right)^{\prime}\left(u_{0}\right), v\right)=\left(\left.\mathcal{A}_{\tau}\right|_{H_{0}}\right)^{\prime}\left(u_{0}\right)(v)=\left(\mathcal{A}_{\tau}^{\prime}\left(u_{0}\right)(v)=\left(\mathcal{A}_{\tau}^{\prime}\left(u_{0}\right), v\right) \forall v \in H_{0} .\right.
$$

Since $\left(\left.\mathcal{A}_{\tau}\right|_{H_{0}}\right)^{\prime}\left(u_{0}\right)=0,\left(\mathcal{A}_{\tau}^{\prime}\left(u_{0}\right), v\right)=0 \forall v \in H_{0}$, i.e., $\mathcal{A}_{\tau}^{\prime}\left(u_{0}\right) \in H_{0}^{\perp}$. It follows that $\mathcal{A}_{\tau}^{\prime}\left(u_{0}\right)=0$. The desired result is proved.

Combing this lemma with Lemma 5 in [1] we have the claim that for every $S \in \mathbb{R}$ there exists a $T>0$ such that

$$
u \in H_{0}, \mathcal{A}_{\tau}^{\prime}(u)=0 \text { and } \mathcal{A}_{\tau}(u) \leq S \Rightarrow \text { either } u(t) \text { is a constant or } \tau \geq T \text {. }
$$

As in [1] we always assume that $\max V=0$ so that $\mathcal{A}_{\tau}(u) \geq 0 \forall u \in H$ and that the ground level zero is attained only at the constant functions that maximize $V$. Then the critical value sets $C_{\tau}=\left\{\mathcal{A}_{\tau}(u) \mid \mathcal{A}_{\tau}^{\prime}(u)=0, u \in H_{0}\right\} \neq \emptyset$ and $C_{\tau} \supset C_{0}=\{-V(z) \mid \nabla V(z)=0\}$ for any $\tau>0$. Let $\bar{H}_{0}=\left\{u \in H_{0} \mid \bar{u}=\right.$ $\left.\int_{0}^{1} u(t) d t=0\right\}$ and

$$
\mathrm{P}: H_{0} \rightarrow \mathbb{T}^{n} \times \bar{H}_{0}, u \mapsto([\bar{u}], u-\bar{u}),
$$

where $[\bar{u}]$ is the equivalence class of $\bar{u} \in \mathbb{R}^{n}$ in $\mathbb{T}^{n}=\mathbb{R}^{n} / \mathbb{Z}^{n}$. Then $\mathrm{P}$ is surjective because $\left\{u \in H_{0} \mid u=\bar{u}\right\}$ is isomorphic to $\mathbb{R}^{n}$ and there exists a 
natural orthogonal decomposition $H_{0}=\mathbb{R}^{n} \oplus \bar{H}_{0}$ given by $u=\bar{u}+(u-\bar{u})$. The Lusternik-Schnirelmann category can be used to associate an index to every closed subset $S$ of $H_{0}$ as follows: $i(S)=$ cat $_{\mathbb{T} n} \times \bar{H}_{0}(\mathrm{P}(S))$. Since cat $\mathbb{T}_{\mathbb{T}} \mathbb{T}^{n}=n+1$ and $\bar{H}_{0}$ is contractible, one has $i\left(H_{0}\right)=n+1$, and $i(S)=n+1$ if and only if $\{[\bar{u}] \mid u \in S\} \equiv \mathbb{T}^{n}$. In particular, $\Gamma=\left\{S \subset H_{0} \mid S\right.$ is closed and $i(S)=$ $n+1\} \neq \emptyset$. The standard arguments (using [3, Prop.1.10]) can prove that $c(\tau):=\inf _{S \in \Gamma} \sup _{S} \mathcal{A}_{\tau}$ is a critical value for $\mathcal{A}_{\tau}$ on $H_{0}$ (cf.[1]). Corresponding to Proposition 6 in [1] we have

Lemma 3. If $V$ is nonconstant, then for every $\tau>0$ there exists a critical value $c(\tau) \in C_{\tau}$ of $\mathcal{A}_{\tau}$ on $H_{0}$ satisfying:

(i) $0<c(\tau) \leq-\min _{\mathbb{R}^{n}} V$ for every $\tau$;

(ii) $\tau \rightarrow c(\tau)$ is decreasing and locally Lipschitz continuous in $(0,+\infty)$;

(iii) $c(\tau) \rightarrow 0$ as $\tau \rightarrow \infty$

Proof The proofs of (i) and (ii) are completely same as that of [1]. (iii) can also be obtained by slightly modifying proof in [1]. The goal is to construct a closed subset $S_{\tau} \in \Gamma$ for each $\tau$ such that $\sup _{S_{\tau}} \mathcal{A}_{\tau} \rightarrow 0$ as $\tau \rightarrow \infty$. For any $\tau$ and $\lambda \in[0,1]$, define $\psi_{\lambda}^{\tau}:[0,1] \rightarrow \mathbb{R}, \quad t \mapsto(1+\tau) \lambda-2 \tau|t-1 / 2|$, then $\psi_{0}^{\tau}(t) \leq 0$ and $\psi_{1}^{\tau}(t) \geq 1$. It follows that maps

$$
\theta_{\lambda}^{\tau}:[0,1] \rightarrow[0,1], t \mapsto \min \left\{1, \max \left\{0, \psi_{\lambda}^{\tau}(t)\right\}\right\}
$$

satisfy: $\theta_{0}^{\tau} \equiv 0, \theta_{1}^{\tau} \equiv 1, \theta_{\lambda}^{\tau}(0)=\theta_{\lambda}^{\tau}(1)$ and $\theta_{\lambda}^{\tau}(t+1 / 2)=\theta_{\lambda}^{\tau}(-t+1 / 2)$ for any $0 \leq t \leq 1 / 2$. The direct computation shows that

$$
\overline{\theta_{\lambda}^{\tau}}=\int_{0}^{1} \theta_{\lambda}^{\tau}(t) d t= \begin{cases}\frac{\left(1+\tau^{2}\right) \lambda^{2}}{2 \tau}, & 0 \leq \lambda \leq \frac{1}{1+\tau} \\ \frac{2(1+\tau) \lambda}{2 \tau}, & \frac{1}{1+\tau}<\lambda \leq \frac{\tau}{1+\tau} \\ 1-\frac{(1+\tau)^{2}(1-\lambda)^{2}}{2 \tau}, & \frac{\tau}{1+\tau}<\lambda \leq 1\end{cases}
$$

So the map $[0,1] \rightarrow[0,1], \lambda \mapsto \overline{\theta_{\lambda}^{\tau}}$ is homotopic to the identity from $[0,1]$ to $[0,1]$. Define

$$
\Theta_{\left(\lambda_{1}, \lambda_{2}, \cdots \lambda_{n}\right)}^{\tau}:[0,1] \rightarrow[0,1]^{n}, t \mapsto\left(\theta_{\lambda_{1}}^{\tau}, \theta_{\lambda_{2}}^{\tau}, \cdots, \theta_{\lambda_{n}}^{\tau}\right)
$$

where $\lambda_{i} \in[0,1], i=1,2, \cdots, n$. Then the properties of $\theta_{\lambda}^{\tau}$ above imply

$$
\Theta_{\left(\lambda_{1}, \lambda_{2}, \cdots \lambda_{n}\right)}^{\tau} \equiv\left(\lambda_{1}, \lambda_{2}, \cdots \lambda_{n}\right) \quad \text { if }\left(\lambda_{1}, \lambda_{2}, \cdots \lambda_{n}\right) \in \partial[0,1]^{n}
$$

and

$$
\Theta_{\left(\lambda_{1}, \lambda_{2}, \cdots \lambda_{n}\right)}^{\tau}(t+1 / 2)=\Theta_{\left(\lambda_{1}, \lambda_{2}, \cdots \lambda_{n}\right)}^{\tau}(-t+1 / 2) \forall 0 \leq t \leq 1 / 2 .
$$


The latter shows that we can 1-periodically extend $\Theta_{\left(\lambda_{1}, \lambda_{2}, \cdots \lambda_{n}\right)}^{\tau}$ to $\mathbb{R}$, still denoted by $\Theta_{\left(\lambda_{1}, \lambda_{2}, \cdots \lambda_{n}\right)}^{\tau}$. Now $\Theta_{\left(\lambda_{1}, \lambda_{2}, \cdots \lambda_{n}\right)}^{\tau} \in H_{0}$, and

$$
f:[0,1]^{n} \rightarrow[0,1]^{n},\left(\lambda_{1}, \lambda_{2}, \cdots \lambda_{n}\right) \mapsto \overline{\Theta_{\left(\lambda_{1}, \lambda_{2}, \cdots \lambda_{n}\right)}^{\tau}}=\left(\overline{\theta_{\lambda_{1}}^{\tau}}, \overline{\theta_{\lambda_{2}}^{\tau}}, \cdots, \overline{\theta_{\lambda_{n}}^{\tau}}\right)
$$

is homotopic to the identity map $[0,1]^{n} \rightarrow[0,1]^{n}$.

Fix a point $\eta=\left(\eta_{1}, \eta_{2}, \cdots, \eta_{n}\right) \in V^{-1}(0)$. Define $S_{\tau}=\left\{\eta+\Theta_{\left(\lambda_{1}, \lambda_{2}, \cdots \lambda_{n}\right)}^{\tau} \mid \lambda_{i} \in\right.$ $[0,1], i=1,2, \cdots, n\}$. Then $S_{\tau}$ is closed in $H_{0}$ and $\mathrm{P}\left(S_{\tau}\right)=B_{1} \times B_{2}$, where

$$
\begin{aligned}
B_{2} & =\left\{\Theta_{\left(\lambda_{1}, \lambda_{2}, \cdots \lambda_{n}\right)}^{\tau}-\overline{\Theta_{\left(\lambda_{1}, \lambda_{2}, \cdots \lambda_{n}\right)}^{\tau}} \mid \lambda_{i} \in[0,1], i=1,2, \cdots, n\right\} \\
B_{1} & =\left\{\left[\eta+\overline{\Theta_{\left(\lambda_{1}, \lambda_{2}, \cdots \lambda_{n}\right)}^{\tau}}\right] \mid \lambda_{i} \in[0,1], i=1,2, \cdots, n\right\} \\
& =\left\{[\eta+\zeta] \mid \zeta \in f\left([0,1]^{n}\right)\right\} \\
& =\left\{[\zeta] \mid \zeta \in f\left([0,1]^{n}\right)\right\}=\mathbb{T}^{n}
\end{aligned}
$$

because $f\left([0,1]^{n}\right)=[0,1]^{n}$ and $\mathbb{T}^{n}=\mathbb{R}^{n} / \mathbb{Z}^{n}=[0,1]^{n} / \partial[0,1]^{n}$. From $\bar{H}_{0}$ being contractible, it follows that $i\left(S_{\tau}\right)=\operatorname{cat}_{\mathbb{T}^{n} \times \bar{H}_{0}} B_{1} \times B_{2}=\operatorname{cat}_{\mathbb{T} n} B_{1}=n+1$. Hence $S_{\tau} \in \Gamma$. Repeating the arguments at the end of proof of Proposition 6 in [1], one can prove that $S_{\tau}$ satisfies our requirements, that is, $\sup _{S_{\tau}} \mathcal{A}_{\tau} \rightarrow 0$ as $\tau \rightarrow \infty$.

Lemma 4([1, Prop.12]). Assume that $M$ is a real analytic manifold, $\varphi$ : $M \rightarrow \mathbb{R}$ is a real analytic function, $C=\left\{x \in M \mid \varphi^{\prime}(x)=0\right\}$ and $\varphi^{\prime \prime}(x)$ is a Fredholm map for every $x \in C$. Then $\varphi$ is locally constant on $C$. Hence, if $K$ is a compact subset of $C$, then $\varphi(K)$ is finite.

Now we are a position to give a corresponding result with Proposition 8 in [1].

Lemma 5. Under the assumptions that $A$ and $V$ are analytic, it holds that

(i) $C_{0}$ is finite;

(ii) $C_{\tau}$ is discrete for any $\tau>0$;

(iii) zero is isolated in $\cup_{0<s<\tau_{0}} C_{s}$ for every $\tau_{0}>0$.

Let $C=\left\{q \in \mathbb{R}^{n} \mid V^{\prime}(q)=0\right\}$ and $K=C \cap[0,1]^{n}$. Applying Lemma 4 to $V$, one gets $V(K)=V(C)=-C_{0}$ is finite. (ii) and (iii) can be proved with (5) and Lemma 4 as in $[1, \S 5]$.

Finally, repeating the proof of $[1$, Theorem 1] we can immediately derive Theorem 1 from Lemma 3 and Lemma 5 above. 


\section{References}

[1] J. Campos and M. Tarallo, Large minimal period orbits of periodic autonomous system, Nonlinearity, 17 (2004), 357-370.

[2] Yiming Long, Guangcun Lu, Infinitely many periodic solution orbits of autonomous Lagrangian systems on tori, J. Funct. Anal., 197(2003), 301322.

[3] P.H. Rabinowitz, On a class of functionals invariant under a $\mathbb{Z}^{n}$ action, Trans. Amer. Math. Soc., 310(1988), no. 1, 303-311.

[4] H. Seifert, Periodische Bewegungen Mecanischer System, Math. Z., 51(1948), 197-216.

Received: May 8, 2007 